\title{
Vasopressin, but not oxytocin, increases empathic concern among individuals who received higher levels of paternal warmth: A randomized controlled trial
}

\author{
Benjamin A. Tabak ${ }^{a, *}$, Meghan L. Meyer ${ }^{a}$, Elizabeth Castle ${ }^{a}$, \\ Janine M. Dutcher ${ }^{a}$, Michael R. Irwin ${ }^{a, b, c, d}$, Jung H. Han ${ }^{a}$, \\ Matthew D. Lieberman ${ }^{a, b}$, Naomi I. Eisenberger ${ }^{a}$
}

\author{
a Department of Psychology, University of California - Los Angeles, CA, United States \\ b Department of Psychiatry and Biobehavioral Sciences, David Geffen School of Medicine, University of \\ California - Los Angeles, CA, United States \\ c Cousins Center for Psychoneuroimmunology, David Geffen School of Medicine, University of California - \\ Los Angeles, CA, United States \\ d Semel Institute for Neuroscience, David Geffen School of Medicine, University of California - \\ Los Angeles, CA, United States
}

Received 11 July 2014; received in revised form 7 October 2014; accepted 7 October 2014

\section{KEYWORDS \\ Vasopressin; \\ Oxytocin; \\ Empathic concern; \\ Paternal warmth; \\ Intranasal \\ administration}

\begin{abstract}
Summary
Background: Empathy improves our ability to communicate in social interactions and motivates prosocial behavior. The neuropeptides arginine vasopressin and oxytocin play key roles in socioemotional processes such as pair bonding and parental care, which suggests that they may be involved in empathic processing.

Methods: We investigated how vasopressin and oxytocin affect empathic responding in a randomized, double-blind, placebo controlled, between-subjects study design. We also examined the moderating role of parental warmth, as reported in the early family environment, on empathic responding following vasopressin, oxytocin, or placebo administration.

Results: Among participants who reported higher levels of paternal warmth (but not maternal warmth), vasopressin (vs. placebo and oxytocin) increased ratings of empathic concern after viewing distressing and uplifting videos. No main or interaction effects were found for individuals who received oxytocin.
\end{abstract}

* Corresponding author at: Department of Psychology, UCLA, 1285 Franz Hall, Box 951563, Los Angeles, CA 90095-1563, United States. Tel.: +1 3108252961 .

E-mail address: btabak@psych.ucla.edu (B.A. Tabak). 
Conclusions: Vasopressin has a role in enhancing empathy among individuals who received higher levels of paternal warmth.

Trial registration: NCT01680718.

(c) 2014 Elsevier Ltd. All rights reserved.

Empathy improves our ability to communicate in social interactions and motivates prosocial behavior (Batson et al., $1987,1988)$. While a great deal of psychological research has shown the social importance of empathy, far less is known about the neurobiological processes that influence empathy. Recent research has examined the role of the neuropeptide oxytocin (OT) in empathy due to its involvement in many socioemotional processes (Bartz et al., 2011). Studies investigating the effects of OT on empathy in healthy individuals have produced mixed results. Initial evidence suggested no effect (Singer et al., 2008), but more recent work has shown that empathy does increase following OT administration (Abu-Akel et al., 2014; Shamay-Tsoory et al., 2013). Other studies suggest that OT may influence specific components of empathy (Hurlemann et al., 2010; Theodoridou et al., 2013) and that the effect of OT on empathy may be moderated by individual differences (Bartz et al., 2010a,b).

Less work has explored the role of the neuropeptide arginine vasopressin (AVP; which is structurally similar to OT and diverges by two amino acids; Gimpl and Fahrenholz, 2001) in empathy. In addition to AVP's function in vasoconstriction and water retention, AVP plays a key role in pair bond formation, parental care, and social approach in a variety of species (Goodson, 2013; Lim and Young, 2006) and thus may influence empathic responses. In a recent study of the social monogamous coppery titti monkey, AVP V1a receptor binding was wide-spread throughout the brain, including the cingulate and insular cortices (Freeman et al., 2014a,b), which are implicated in emotional empathy (Shamay-Tsoory, 2011). Additionally, in monogamous male prairie voles, an AVP $\mathrm{V} 1 \mathrm{a}$ receptor antagonist reduced affiliative and attachment behavior (Winslow et al., 1993). In humans, variation in the AVP V1a receptor gene has been associated with prosociality (Avinun et al., 2011; Knafo et al., 2008), including partner bonding in married couples (Walum et al., 2008). Variation in AVP V1a receptors has also been associated with autism spectrum disorders, a neurodevelopmental disorder characterized by social impairments including deficits in empathy (Kim et al., 2002; Wassink et al., 2004; Yirmiya et al., 2006). In addition, studies of heterosexual couples have found positive associations between plasma AVP and indices of positive relationship functioning as well as decreased negative communication behaviors (Gouin et al., 2010, 2012, but see Taylor et al., 2010).

Although AVP is hypothesized to contribute to the neurobiological mechanisms underlying empathy, little experimental research in humans has examined the effect of AVP on empathic responding. Empathy is typically decomposed into two components: (1) cognitive empathy, which refers to the ability to understand others' thoughts and to take their perspective and (2) emotional or affective empathy, which consists of empathic concern, or feelings of concern and warmth toward others, along with personal distress that involves experiencing stress and anxiety as a result of another's suffering (Batson et al., 1987; ShamayTsoory, 2011). Although research has shown that AVP does not seem to improve cognitive empathy (Kenyon et al., 2013; Uzefovsky et al., 2012), less work has examined affective empathy. Given that empathic concern, one component of affective empathy, is the primary motivator of prosocial behavior (Batson et al., 1988, 1987) and that multiple studies have shown that AVP increases human prosocial behavior (Rilling et al., 2012, 2014), AVP may have specific effects on empathic concern.

Another factor that may be important to consider in understanding the effect of AVP and OT on empathic concern is the role of the early environment. For example, research on OT has shown that its effects on human prosociality are sensitive to early environmental factors. Recent studies have demonstrated that the early family environment, and specifically a positive family environment, appears to make people more susceptible to OT's effects on prosocial behavior often motivated by empathy (Riem et al., 2013; Van IJzendoorn et al., 2011). For example, in an all female sample, Van IJzendoorn et al. (2011) found that OT increased charitable giving, but only among those who reported having more supportive parents.

Given the role of AVP and OT in socioemotional processing, and recent findings demonstrating that positive early family environments may increase susceptibility to the effects of OT on prosociality (e.g., Riem et al., 2013; Van IJzendoorn et al., 2011), we investigated the interaction between drug condition (AVP, OT, and placebo) and an aspect of the family environment that has been shown to affect empathy development: reported experiences of parental warmth (Zhou et al., 2002). In addition, based on previous studies demonstrating sexually dimorphic effects of AVP and OT (Rilling et al., 2012, 2014; Thompson et al., 2006), we included a sample of women and men. We hypothesized that AVP and OT would increase empathic concern compared to placebo, but only among individuals who received higher levels of parental warmth.

\section{Materials and methods}

\subsection{Participants}

Participants were 125 undergraduate students from the University of California, Los Angeles (90 female, age range $=18-31$ years, Mean age $=20.88, S D=2.71$ ). They were randomly assigned to receive intranasal AVP $(n=42 ; 30$ female, 12 male), OT ( $n=42$; 30 female, 12 male) or placebo $(n=41 ; 30$ female, 11 male). Exclusion criteria included current allergies or cold symptoms as well as present or history of heart disease, hypertension, myocardial infarction, cardiac arrhythmia, kidney or liver disease, vascular disease, epilepsy, migraine, asthma, nephritis, diabetes or another 
endocrine disease, frequent or unexplained fainting, stroke, aneurysm or brain hemorrhage, other neurological illness, or current restricted fluid intake. Exclusion criteria also included use of current mediations (e.g., vasoconstrictive medications), present psychiatric diagnosis including alcohol abuse, pregnancy, breastfeeding, and smoking $>15$ cigarettes per day. Participants were asked to refrain from using medication or alcohol for $24 \mathrm{~h}$, caffeine for $4 \mathrm{~h}$, and food or drinks (except water) for $2 \mathrm{~h}$ preceding the experiment (see Supplementary Fig. 1 for more detailed information). The sample consisted of participants who selfidentified as Asian (56.0\%), White (20.8\%), Hispanic/Latino (13.6\%), Black or African American (4.8\%), and "Other" (4.8\%). $11.4 \%$ identified as Hispanic/Latino in addition to one of the groups described above (except for those who only identified as Hispanic/Latino). Participants who completed all aspects of the study were paid $\$ 40-\$ 50$ depending on their choices in another task not relevant to the present study. Informed consent was obtained from all participants and the UCLA Institutional Review Board approved this study.

\subsection{Procedure}

Each participant completed two sessions. In the first session, participants completed several self-report questionnaires that included the assessment of parental warmth using the Parental Bonding Instrument (PBI; Parker et al., 1979; described below).

In the second session (completed on average 18.36 days after the first session, $S D=16.08$ ) participants arrived in groups of $2-15$ at a computer lab where they each had their own independent computer. All participants completed the second session between the hours of 2:00 pm and 5:30 pm. Participants first completed a set of questionnaires pre-administration including measures of positive and negative affect using the Positive and Negative Affect Schedule (PANAS; Thompson, 2007; described below). All participants also provided a urine sample, which was tested for possible pregnancy and drug use. Research nurses then checked all participants' temperature, heart rate, and blood pressure to ensure that they were in the accepted limits: systolic blood pressure between 90 and 130, diastolic blood pressure between 60 and 90, heart rate between 55 and 100 beats $/ \mathrm{min}$, and temperature less than $100^{\circ} \mathrm{F}$. If vital signs were out of range, participants rested for $10-15 \mathrm{~min}$ and measurements were repeated until readings were within acceptable limits; one participant was excluded on basis of abnormal vital signs and did not receive AVP, OT, or placebo.

In preparation for each drug-administration session, a third-party research coordinator unrelated to the present study used an online random number generator (www.random.org) to randomly assign participants to the AVP, OT, or placebo condition (blocked on gender; allocation ratio of $1: 1)$ and communicated this information to the UCLA pharmacy. A UCLA pharmacist prepared the drug or placebo for each participant; there was no indication on the label as to what was received (to maintain the blind). Approximately $1 \mathrm{~h}$ after arriving, participants received AVP, OT, or placebo using a randomized, double-blind, placebocontrolled, between-subjects procedure. We used sterile $6 \mathrm{ml}$ amber glass bottles with metered nasal pumps from Advantage Pharmaceuticals, Inc. Participants first received instructions on how to use the nasal sprays from the first author and a UCLA research nurse. Participants were then instructed to deliver one spray per nostril in an alternating fashion when prompted (every $30 \mathrm{~s}$ ).

AVP was provided by American Regent Laboratories, Shirley, NY, USA. The pharmacist transferred AVP $(20 \mathrm{lU} / \mathrm{ml})$ into the bottles with attached intranasal applicators (1 puff $=0.1 \mathrm{ml}$ ). Participants self-administered 5 puffs per nostril (2IU/puff) for a total dose of $20 \mathrm{IU}$. AVP has been shown to exert effects on behavior at this dosage (e.g., Rilling et al., 2012, 2014; Thompson et al., 2006; Uzefovsky et al., 2012). OT (Syntocinin) was provided by Novartis Pharmaceuticals, Switzerland. The pharmacist transferred OT $(24 \mathrm{IU} / \mathrm{ml})$ into the bottles with attached intranasal applicators ( 1 puff $=0.1 \mathrm{ml}$ ). Participants self-administered 5 puffs per nostril (2.4 IU/puff) for a total dose of $24 \mathrm{IU}$. Administration of $24 \mathrm{IU}$ of OT has also been shown to affect behavior in previous studies (e.g., Bartz et al., 2010a,b; Rilling et al., 2012, 2014). Placebo (used previously by Rilling et al., 2014) consisted of $2 \mathrm{ml}$ glycerine and $3 \mathrm{ml}$ purified water (methylparaben and propylparaben mixed according to purified water formula) for a total of $5 \mathrm{ml}$. This was filtered with a $5 \mu \mathrm{m}$ filter and transferred to the bottles with attached intranasal applicators $(1$ puff $=.1 \mathrm{ml})$. Participants self-administered 5 puffs per nostril.

As in previous research (Rilling et al., 2012, 2014), following completion of administration, participants waited approximately $40 \mathrm{~min}$ before beginning the tasks. During this time, participants were asked to sit quietly and read from a stack of 10 magazines (e.g., Newsweek). They were also instructed to turn off their phones and refrain from speaking to one another. Participants then completed the PANAS (Thompson, 2007). Next, they completed a series of tasks unrelated to empathy that were presented in randomized order to prevent order effects (e.g., working memory, deception detection, perceptions of faces). The present study is focused on the empathy task (described below). Study personnel and research nurses were blind to the drug condition (there were participants in each condition in each group session). The first author supervised the procedure and was present throughout every session.

To induce empathy, we used two videos provided by Sze et al. (2012) that were presented in random order. The first video, referred to hereafter as the "distressing video"' (117s), began with a short introduction to the crisis in Darfur and followed with images of the horrible conditions experienced by individuals living in the region. This included images of children and adults who were receiving aid because they were sick, wounded, and/or emaciated. The second video, referred to hereafter as the "uplifting", video $(116 \mathrm{~s})$, began with a short introduction to autism spectrum disorders and followed with uplifting images from Surfers Healing, a non-profit organization that teaches children with autism how to swim and surf. Immediately upon finishing each video, participants responded to the questions described below that assessed levels of empathic concern.

\subsection{Measures}

\subsubsection{Maternal and paternal warmth}

Participants completed the Mother and Father Care subscale of the PBI (Parker et al., 1979) in a separate session 
Table 1 Means and standard deviations of positive and negative affect.

\begin{tabular}{llll}
\hline & AVP & OT & Placebo \\
\hline Pre-administration positive affect & $2.54(.92)$ & $2.62(.89)$ & $2.63(.91)$ \\
Post-administration positive affect & $2.00(.79)$ & $2.06(.75)$ & $2.03(.76)$ \\
Pre-administration negative affect & $1.30(.35)$ & $1.14(.19)$ & $1.23(.33)$ \\
Post-administration negative affect & $1.12(.24)$ & $1.08(.15)$ & $1.15(.29)$ \\
\hline
\end{tabular}

that occurred on a day prior to the drug administration. The Care subscale of the $\mathrm{PBI}$ asks participants to rate how warm and affectionate their mother and father were during childhood up until age 16. The two 12-item measures (one for mother and one for father) are rated using a 4point Likert-type scale ( $0=$ very unlike; $3=$ very like) and include questions such as, "Spoke to me in a warm and friendly voice," and "Was affectionate to me." Six participants either did not have a father, or did not choose to rate their father's level of paternal warmth. A mean composite was created to represent maternal $(\alpha=.89)$ and paternal warmth $(\alpha=.88)$. Both measures of parental warmth were mean centered in all analyses. Importantly, there were no differences between the AVP, OT, and placebo groups on the two subscales ( $p s>.3$ ).

\subsubsection{State empathic concern}

Immediately after watching each video, participants rated the extent to which they felt empathic concern based on three adjectives (sympathetic, moved, compassionate) used in previous research (Sze et al., 2012) using a 5-point Likerttype scale ( $1=$ not at all; $5=$ extremely). A mean composite score was created to represent empathic concern after watching the distressing video $(\alpha=.84)$ and uplifting video $(\alpha=.84)$

\subsubsection{State positive and negative affect}

To examine whether self-reported positive and/or negative affect changed due to AVP or OT administration, participants completed the 10-item PANAS (Thompson, 2007) pre-administration and approximately $40 \mathrm{~min}$ postadministration before beginning the task. Items on the PANAS are rated using a 5-point Likert-type scale $(1=$ not at all; 5=extremely). A mean composite was created to represent positive affect (pre-administration $\alpha=.82$; post-administration $\alpha=.83$ ) and negative affect (preadministration $\alpha=.6$; post-administration $\alpha=.63$ ). Change scores (post-administration - pre-administration) were then computed to examine differences in positive and negative affect before and after drug-administration.

\subsection{Statistical analysis}

We first conducted Analyses of Variance (ANOVA) to examine non-specific drug effects (i.e., main effects of pre-post changes in positive and negative affect) as well as the main effect of drug condition (AVP, OT, placebo) on empathic concern. We then conducted Analyses of Covariance (ANCOVA) to examine the interaction between drug condition (AVP, OT, placebo) and maternal warmth as well as drug condition and paternal warmth on empathic concern. Significant omnibus interactions were further investigated through the use of hierarchical linear regression analysis that examined differences between two drug conditions at a time (i.e., AVP vs. placebo, OT vs. placebo, and AVP vs. OT). Post hoc tests of specific interaction effects were conducted using PROCESS (Hayes, 2013). p-values <.05 (two-tailed) were considered statistically significant. One participant unintentionally began completing post-administration questionnaires and tasks approximately 25-min post-administration instead of approximately 40 min post-administration. Results remained unchanged when removing this participant from analyses. Additionally, all results remained unchanged when removing outliers on all major study variables based on scores lower than the 25 th percentile $-1.5 \times$ the interquartile range and scores higher than the 75th percentile $+1.5 \times$ the interquartile range. For this reason, all data is included in the results reported. In all analyses, the dependent variable was a continuous measure of empathic concern following each video. Measures of maternal warmth, paternal warmth, and empathic concern were continuous. All analyses were conducted using SPSS version 20. Figures were created using Stata version 13. Based on the moderate to large main effect (Cohen's $D=0.7$ ) of AVP on a different type of empathic processing (i.e., emotion recognition; Uzefovsky et al., 2012), we estimated that we would need at least $n=34$ participants in each group to achieve $>0.8$ power ( $G$ *Power 3.1).

\section{Results}

To ensure that any observed effects of drug condition were not simply due to changes in positive or negative affect, we first assessed non-specific main effects of drug condition (AVP, OT, placebo) on changes in positive and negative affect. No differences were found between drug conditions on pre-post changes in positive, $F(2,122)=.05, p=.95$, or negative affect, $F(2,122)=1.73, p=.18$ (See Table 1 for group means and SDs).

With regard to the videos, there was a main effect of video type (distressing $[M=3.21, S D=1.12]$ vs. uplifting $[M=2.59, S D=1.03]$ ) on empathic concern, $t(124)=7.97$, $p<.001$, such that the distressing video elicited higher levels of empathic concern compared to the uplifting video. Hence, the effects of drug condition and parental warmth on empathic concern were analyzed separately for the two videos. There were no interactions between video type and drug condition, $F(2,122)=.16, p=.85$ (see Table 2 for group means and SDs). 
Table 2 Means and standard deviations of empathic concern.

\begin{tabular}{lll}
\hline & Uplifting & Distressing \\
\hline AVP & $2.72(1.05)$ & $3.35(1.06)$ \\
OT & $2.51(1.05)$ & $3.17(1.11)$ \\
Placebo & $2.55(1.01)$ & $3.11(1.19)$ \\
\hline
\end{tabular}

\subsection{Effect of drug and parental warmth on empathic concern: distressing video}

For the distressing video, there was no main effect of drug condition on empathic concern, $F(2,122)=.532, p=.589$. However, there was a significant main effect of paternal warmth on empathic concern, $F(1,113)=5.41, p=.022$, $\eta_{\mathrm{p}}{ }^{2}=.05$, as well as a significant interaction between drug condition and paternal warmth on empathic concern, $F(2$, $113)=3.38, p=.037, \eta_{p}{ }^{2}=.06$. There was no main effect of maternal warmth, $F(1,119)=1.5, p=.223$, and no interaction between drug condition and maternal warmth on empathic concern, $F(2,119)=.113, p=.894$.

To further investigate the significant interaction between drug condition and paternal warmth, we conducted post hoc hierarchical linear regression analyses to examine the differences between two drug conditions at a time. We first examined AVP vs. placebo (dummy coded $A V P=1$; placebo $=0$ ) and found a significant interaction between drug condition and paternal warmth on empathic concern, $b=.12, S E=.05, p=.015, R^{2}=.18$, Interaction effect $R^{2}$ change $=.07$. Analysis of simple slopes identified a significant association between paternal warmth and empathic concern in the AVP group, $b=.13, S E=.03, p<.001, R^{2}=.34$, but not in the placebo group, $b=.01, S E=.04, p=.712$. To further explore the significant interaction, we examined mean differences in empathic concern between the AVP and placebo groups at one SD above and below the mean of paternal warmth. There was a significant difference in empathic concern between the AVP and placebo group at higher levels of paternal warmth $(+1 S D), b=.74$, $S E=.32, p=.025$, but no significant difference at lower levels ( $-1 S D), b=-.4, S E=.32, p=.219$ (see Fig. 1). Thus, among individuals who experienced higher levels of paternal warmth in childhood, AVP increased empathic concern. However, AVP did not have a significant effect on empathic concern for individuals who experienced lower levels of paternal warmth.

Next, we examined OT vs. placebo (dummy coded OT = 1 ; placebo $=0$ ) and found no interaction between drug condition and paternal warmth on empathic concern, $b=.002$, $S E=.06, p=.979$. Last, we examined AVP vs. OT (dummy coded $A V P=1 ; O T=0$ ) and found a significant interaction between drug condition and paternal warmth on empathic concern, $b=.12, S E=.06, p=.047, R^{2}=.18$, Interaction effect $R^{2}$ change $=.04$. Analysis of simple slopes identified a significant association between paternal warmth and empathic concern in the AVP group, $b=.13, S E=.03$, $p<.001, R^{2}=.34$, but not in the OT group, $b=.02, S E=.05$, $p=.766$. Further analyses revealed that there was a significant difference in empathic concern between the AVP and OT group at higher levels of paternal warmth $(+1 S D), b=.71$,

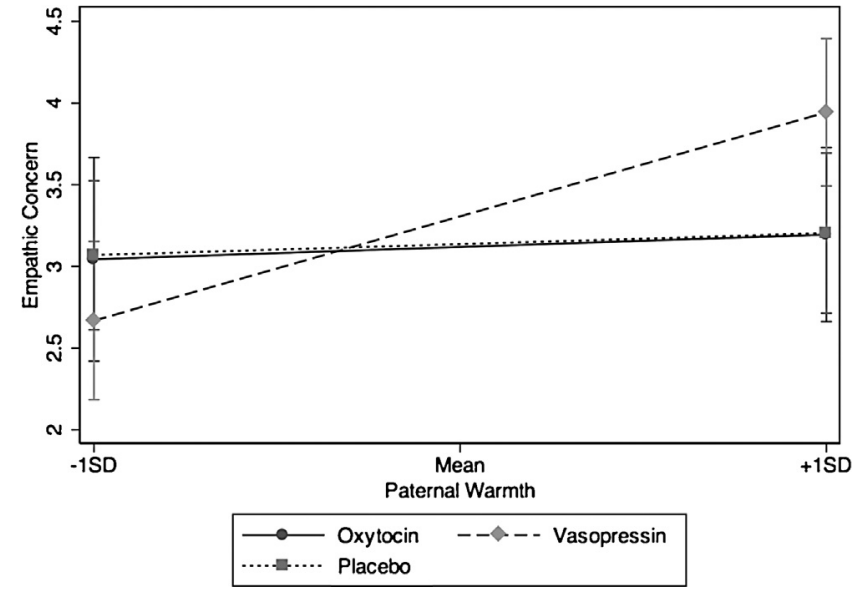

Figure 1 There was a significant interaction of drug condition (AVP, OT, placebo) $\times$ paternal warmth on empathic concern following the distressing video. At $+1 S D$ above the mean of paternal warmth, individuals on AVP reported significantly higher levels of empathic concern compared to those on placebo or OT. Error bars represent $95 \% \mathrm{Cls}$.

$S E=.33, p=.03$, but no significant difference at lower levels $(-1 S D), b=-.24, S E=.33, p=.464$ (see Fig. 1). Thus, among individuals who experienced higher levels of paternal warmth in childhood, AVP increased empathic concern compared to OT. However, AVP (vs. OT) did not have a significant effect on empathic concern for individuals who experienced lower levels of paternal warmth.

\subsection{Effect of drug and parental warmth on empathic concern: uplifting video}

For the uplifting video, there was no effect of drug condition on empathic concern, $F(2,122)=.5, p=.61$. However, similar to the distressing video, there was a significant main effect of paternal warmth on empathic concern, $F(1$, $113)=7.68, p=.007, \eta_{p}{ }^{2}=.06$, as well as a significant interaction between drug condition and paternal warmth on empathic concern, $F(2,113)=3.3, p=.04, \eta_{p}{ }^{2}=.06$. There was no main effect of maternal warmth, $F(1,119)=2.62$, $p=.108$, and no interaction between drug condition and maternal warmth on empathic concern, $F(2,119)=.16$, $p=.853$.

To further investigate the significant interaction between drug condition and paternal warmth, we conducted post hoc hierarchical linear regression analyses to examine the differences between two drug conditions at a time. We first examined AVP vs. placebo (dummy coded AVP=1; placebo $=0$ ) and, like the results from the distressing video, found a significant interaction between drug condition and paternal warmth on empathic concern, $b=.1, S E=.04$, $p=.03, R^{2}=.21$, Interaction effect $R^{2}$ change $=.05$. Analysis of simple slopes identified a significant association between paternal warmth and empathic concern in the AVP group, $b=.13, S E=.03, p<.001, R^{2}=.35$, but not in the placebo group, $b=.04, S E=.03, p=.273$. To further explore the significant interaction, we examined mean differences in empathic concern between the AVP and placebo groups at one $S D$ above and below the mean of paternal warmth. 


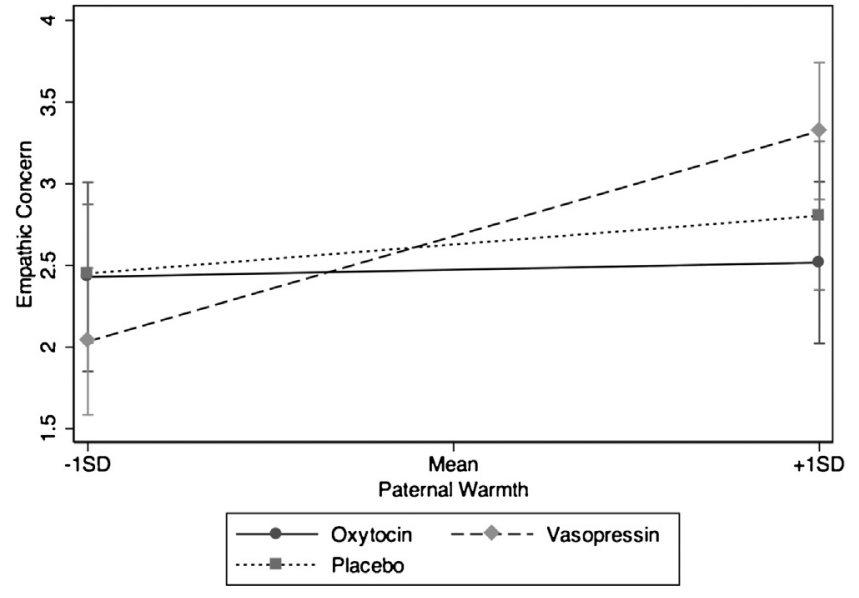

Figure 2 There was a significant interaction of drug condition (AVP, OT, placebo) $\times$ paternal warmth on empathic concern following the uplifting video. At $+1 S D$ above the mean of paternal warmth, individuals on AVP reported significantly higher levels of empathic concern compared to those on OT and marginally higher levels of empathic concern compared to those on placebo. Error bars represent $95 \% \mathrm{Cls}$.

There was a marginally significant difference in empathic concern between the AVP and placebo group at higher levels of paternal warmth $(+1 S D), b=.52, S E=.3, p=.086$, but no significant difference at lower levels ( $-1 S D), b=-.42$, $S E=.3, p=.17$ (see Fig. 2). Thus, among individuals who experienced higher levels of paternal warmth in childhood, AVP marginally increased empathic concern to the uplifting video. However, AVP did not have a significant effect on empathic concern for individuals who experienced lower levels of paternal warmth.

Comparing OT with placebo (dummy coded OT=1; placebo $=0$ ) revealed no interaction between drug condition and paternal warmth on empathic concern, $b=-.03$, $S E=.06, p=.643$. Last, we examined AVP vs. OT (dummy coded $A V P=1$; OT $=0$ ) and again found a significant interaction between drug condition and paternal warmth on empathic concern, $b=.12, S E=.06, p=.028, R^{2}=.19$, Interaction effect $R^{2}$ change=.05. Analysis of simple slopes identified a significant association between paternal warmth and empathic concern in the AVP group, $b=.13, S E=.03$, $p<.001, R^{2}=.35$, but not in the OT group, $b=.01, S E=.05$, $p=.856$. Further analyses revealed that there was a significant difference in empathic concern between the AVP and OT group at higher levels of paternal warmth (+1 SD), $b=.77, S E=.31, p=.015$, but not at lower levels $(-1 S D)$, $b=-.25, S E=.32, p=.43$ (see Fig. 2). Thus, among individuals who experienced higher levels of paternal warmth in childhood, AVP increased empathic concern compared to OT. However, AVP (vs. OT) did not have a significant effect on empathic concern for individuals who experienced lower levels of paternal warmth.

\subsection{Video order effects}

Videos were presented in random order, but to ensure that the significant interaction between drug condition and paternal warmth was not affected by the video order, an
ANCOVA was conducted to examine the three-way interaction between drug condition, paternal warmth, and video order. There was no interaction with video order on empathic concern in the distressing video, $F(2,107)=2.36$, $p=.1$, or in the uplifting video, $F(2,107)=2.19, p=.803$. Thus, video order did not appear to affect the present results.

\subsection{Gender effects}

Due to potential sexually dimorphic effects of AVP and OT (Rilling et al., 2012, 2014), we also examined the effect of participant gender on empathic concern following each of the videos. For the distressing video, an ANCOVA with drug condition (AVP, OT, placebo) and gender as betweensubjects factors and paternal warmth as a covariate showed no main effect of gender, $F(1,107)=.24, p=.623$, no twoway interactions ( $p s>.15)$, and no three-way interaction, $F(2,107)=.237, p=.789$. An ANCOVA with drug condition and gender as between-subjects factors and maternal warmth as a covariate showed no main effect of gender, $F(1,113)=.65$, $p=.423$, no two-way interactions ( $p s>.8$ ) and no three-way interaction, $F(2,113)=1.95, p=.147$.

For the uplifting video, An ANCOVA with drug condition and gender as between-subjects factors and paternal warmth as a covariate showed no main effect of gender, $F(1$, $107)=.007, p=.931$, no two-way interactions ( $p s>.33$ ), and no three-way interaction, $F(2,107)=.2, p=.822$. Likewise, an ANCOVA with drug condition and gender as betweensubjects factors and maternal warmth as a covariate showed no main effect of gender, $F(1,113)=.034, p=.854$, no twoway interactions ( $p s>.45)$ and no three-way interaction, $F(2,113)=.62, p=.541$. Because none of the interactions that included gender were significant, gender was not included as a covariate in the analyses reported (see Supplementary Material for gender-specific analyses).

\section{Discussion}

Our findings provide the first evidence that childhood experiences of paternal warmth moderate the effect of AVP on empathic concern. Specifically, AVP increased empathic concern in a sample of female and male participants who experienced higher levels of paternal warmth in childhood. This effect was not found for OT and it was not gender specific. In addition, this effect was observed for both the distressing and the uplifting videos, suggesting that these effects extend across empathy for the negative experiences of others (e.g., those suffering in Darfur) as well as empathy for the accomplishments made by others (e.g., individuals with autism learning how to swim and surf).

Studies have shown that parental care, or a lack thereof, can alter AVP expression and immunoreactivity in several animal species (Bester-Meredith and Marler, 2003; Frazier et al., 2006; Veenema and Neumann, 2009). Francis et al. (2002) found that differences in rat maternal care (i.e., high or low licking and grooming) were associated with increased oxytocin receptor binding in female (but not male) adult offspring and increased AVP V1a receptor binding in male (but not female) adult offspring. In addition, in the monogamous prairie vole (who, like humans, show biparental care of 
offspring), central administration of AVP promotes (whereas an AVP antagonist reduces) parental behavior in males but not females (for review see, Wang et al., 1999). That the moderation of AVP's effects on empathic concern in the present study was specific to paternal warmth, as opposed to maternal warmth, suggests that human paternal parenting style may have specific effects on epigenetic modification of AVP V1a receptors. However, the mechanism through which this might occur is not yet known as limited research has examined how paternal behavior affects the vasopressin system in female offspring and the function of AVP V1a receptors in females are less well understood (Bales et al., 2007). Future work is needed to examine these processes.

To date, research investigating the effects of AVP and OT on empathy has produced inconsistent results. For example, in one study AVP reduced cognitive empathic ability (i.e., the ability to recognize negative emotions; Uzefovsky et al., 2012), but Kenyon et al. (2013) found no effect of AVP on cognitive empathy. Singer et al. (2008) found no effect of OT on affective empathy whereas others found increased empathy following OT administration (Abu-Akel et al., 2014; Shamay-Tsoory et al., 2013). OT has also been found to increase affective, but not cognitive empathy (Hurlemann et al., 2010), as well as cognitive, but not affective empathy (Theodoridou et al., 2013). The present results are in agreement with Singer et al. (2008) and Theodoridou et al. (2013) and found no main effect of OT on affective empathy. However, based on recent evidence demonstrating that the effect of OT on prosocial behavior is specific to individuals who have more positive family environments in general (Van IJzendoorn et al., 2011), we hypothesized that parental warmth would moderate the effect of AVP and OT on empathy.

Bartz et al. (2010a,b) found that the effect of OT administration on recollections of maternal care and closeness was moderated by attachment anxiety, which may reflect early negative interactions with a caregiver. Specifically, following OT administration individuals with lower levels of attachment anxiety remembered their mothers as more close and caring, whereas individuals with higher levels of attachment anxiety remembered their mothers as less caring (Bartz et al., 2010a,b). The study by Bartz et al. (2010a,b) suggested that maternal parenting style may have more specific effects on the OT system, but the present results do not confirm this finding. One possibility for the discrepancy in results is that both the moderator and outcome variables examined in Bartz et al. (2010a,b) differ compared to the present study. It is possible that adult attachment anxiety and experiences of parental warmth in childhood and adolescence may moderate the effects of OT differently.

In addition to demonstrating the moderating role of paternal warmth on AVP's effects, our results add to studies identifying the prosocial influence of AVP in animals (Lim and Young, 2006; Winslow et al., 1993) as well as human men (Rilling et al., 2012) and women (Rilling et al., 2014; Thompson et al., 2006). Our findings also add to the smaller number of studies that have administered AVP and OT to females and shown prosocial effects (e.g., Rilling et al., 2012, 2014). Although OT is often considered more relevant for females and AVP more relevant for males (Carter, 2007), both play a significant role in regulating socioemotional processes in males and females (Cushing and Kramer, 2005; Insel and Young, 2001) and warrant additional attention in future research. This is made apparent most recently by the work of Rilling and colleagues who found sexually dimorphic effects of OT and AVP at the behavioral and neural level (2012, 2014).

Results from the present study also contribute to a more nuanced understanding of the effects of neuropeptides on socioemotional processes. Indeed, just as research continues to identify individual difference factors and contextual variables that moderate the effects of intranasal OT (Bartz et al., 2011; Macdonald, 2012), as well as associations between variation in oxytocin receptor gene polymorphisms (Tabak, 2013), the effects of AVP also appear to be moderated by similar factors (Rilling et al., 2012, 2014; Shalev et al., 2011; Thompson et al., 2006). In addition to our focus on affective rather than cognitive empathy, another potential reason that the present results differ from previous findings (Kenyon et al., 2013; Hurlemann et al., 2010; Theodoridou et al., 2013; Uzefovsky et al., 2012) may relate to our investigation of the moderating role of the early family environment, which was not considered in previous studies focusing on empathy.

Although no gender effects were found in the present study, it is important to note that the majority of our sample was female by a ratio of approximately $3: 1$. Future studies would benefit from including an equal number of men and women to further disentangle potential sexually dimorphic effects of AVP and OT. Based on the work of Born et al. (2002) demonstrating that AVP in cerebrospinal fluid increases after intranasal administration, the assumption in the field is that AVP affects behavior via central mechanisms. However, further research is needed to confirm if and how intranasal AVP is transmitted to the brain (Churchland and Winkielman, 2012; Ludwig et al., 2013). A recent study in rhesus macaques identifying the neuroanatomical distribution of OT receptor binding and mRNA found little OT receptor binding in brain areas associated with empathy (Freeman et al., 2014a,b). Based on this information, it is unlikely that the effects of AVP found in the present study are driven by AVP binding to OT receptors, even though there is known cross-reactivity of AVP with the OT receptor (Koshimizu et al., 2012). Last, our reliance on a retrospective self-report measure of the early family environment is limiting. Future prospective longitudinal research that more precisely measures the childhood environment is needed to confirm the present findings.

In sum, the present findings demonstrate that the effect of AVP, but not OT, on empathic concern is moderated by experiences of paternal warmth in childhood. This novel finding in humans is in agreement with animal research showing that the parental environment can alter the AVP system and contribute to long-term changes in adult behavior (Veenema, 2012; Wang et al., 1999).

\section{Role of the funding source}

Funding was provided by the UCLA Jeffrey/Wenzel Term Chair in Behavioral Neuroscience (to N.I.E.). A postdoctoral fellowship for B.A.T. was supported by the T32MH015750 training fellowship in Biobehavioral Issues in Physical and Mental Health at the University of California, Los Angeles. 


\section{Conflict of interest}

The authors declared that they had no conflict of interest with respect to their authorship or the publication of this article.

\section{Acknowledgements}

We would like to thank Kayla Asato and Zhixing Luo for their assistance in data collection and statistical analysis. We would also like to thank Jocelyn Sze and Robert Levenson for providing us with their video stimuli.

\section{Appendix A. Supplementary data}

Supplementary data associated with this article can be found, in the online version, at http://dx.doi.org/10 1016/j.psyneuen.2014.10.006.

\section{References}

Abu-Akel, A., Palgi, S., Klein, E., Decety, J., Shamay-Tsoory, S., 2014. Oxytocin increases empathy to pain when adopting the other- but not the self-perspective. Soc. Neurosci., 1-9.

Avinun, R., Israel, S., Shalev, I., Gritsenko, I., Bornstein, G., Ebstein, R.P., Knafo, A., 2011. AVPR1A variant associated with preschoolers' lower altruistic behavior. PLoS ONE 6, e25274.

Bartz, J.A., Zaki, J., Bolger, N., Hollander, E., Ludwig, N.N., Kolevzon, A., Ochsner, K.N., 2010a. Oxytocin selectively improves empathic accuracy. Psychol. Sci. 21, 1426-1428.

Bartz, J.A., Zaki, J., Bolger, N., Ochsner, K.N., 2011. Social effects of oxytocin in humans: context and person matter. Trends Cogn. Sci. 15, 301-309.

Bartz, J.A., Zaki, J., Ochsner, K.N., Bolger, N., Kolevzon, A., Ludwig, N., Lydon, J.E., 2010b. Effects of oxytocin on recollections of maternal care and closeness. Proc. Natl. Acad. Sci. U. S. A. 107, 21371-21375.

Batson, C.D., Dyck, J.L., Brandt, J.R., Batson, J.G., Powell, A.L., McMaster, M.R., Griffitt, C., 1988. Five studies testing two new egoistic alternatives to the empathy-altruism hypothesis. J. Pers. Soc. Psychol. 55, 52-77.

Batson, C.D., Fultz, J., Schoenrade, P.A., 1987. Distress and empathy: two qualitatively distinct vicarious emotions with different motivational consequences. J. Pers. 55, 19-39.

Bester-Meredith, J.K., Marler, C.A., 2003. Vasopressin and the transmission of paternal behavior across generations in mated, cross-fostered Peromyscus mice. Behav. Neurosci. 117, 455-463.

Born, J., Lange, T., Kern, W., McGregor, G.P., Bickel, U., Fehm, H.L., 2002. Sniffing neuropeptides: a transnasal approach to the human brain. Nat. Neurosci. 5, 514-516.

Carter, C.S., 2007. Sex differences in oxytocin and vasopressin: implications for autism spectrum disorders? Behav. Brain Res. 176, 170-186.

Churchland, P.S., Winkielman, P., 2012. Modulating social behavior with oxytocin: how does it work? What does it mean? Horm. Behav. 61, 392-399.

Cushing, B.S., Kramer, K.M., 2005. Mechanisms underlying epigenetic effects of early social experience: the role of neuropeptides and steroids. Neurosci. Biobehav. Rev. 29, 1089-1105.

Francis, D.D., Young, L.J., Meaney, M.J., Insel, T.R., 2002. Naturally occurring differences in maternal care are associated with the expression of oxytocin and vasopressin (V1a) receptors: gender differences. J. Neuroendocrinol. 14, 349-353.
Frazier, C.R.M., Trainor, B.C., Cravens, C.J., Whitney, T.K., Marler, C.A., 2006. Paternal behavior influences development of aggression and vasopressin expression in male California mouse offspring. Horm. Behav. 50, 699-707.

Freeman, S.M., Inoue, K., Smith, A.L., Goodman, M.M., Young, L.J., 2014a. The neuroanatomical distribution of oxytocin receptor binding and mRNA in the male rhesus macaque (Macaca mulatta). Psychoneuroendocrinology 45, 128-141.

Freeman, S.M., Walum, H., Inoue, K., Smith, A.L., Goodman, M.M., Bales, K.L., Young, L.J., 2014b. Neuroanatomical distribution of oxytocin and vasopressin 1a receptors in the socially monogamous coppery titi monkey (Callicebus cupreus). Neuroscience 273, 12-23.

Gimpl, G., Fahrenholz, F., 2001. The oxytocin receptor system: structure, function, and regulation. Physiol. Rev. 81, 629-683.

Goodson, J.L., 2013. Deconstructing sociality, social evolution and relevant nonapeptide functions. Psychoneuroendocrinology 38, $465-478$

Gouin, J.-P., Carter, C.S., Pournajafi-Nazarloo, H., Glaser, R., Malarkey, W.B., Loving, T.J., et al., 2010. Marital behavior, oxytocin, vasopressin, and wound healing. Psychoneuroendocrinology 35, 1082-1090.

Gouin, J.-P., Carter, C.S., Pournajafi-Nazarloo, H., Malarkey, W.B., Loving, T.J., Stowell, J., Kiecolt-Glaser, J.K., 2012. Plasma vasopressin and interpersonal functioning. Biol. Psychol. 91, 270-274.

Hayes, A.F., 2013. An Introduction to Mediation, Moderation, and Conditional Process Analysis: A Regression-Based Approach. Guilford Press, New York.

Hurlemann, R., Patin, A., Onur, O.A., Cohen, M.X., Baumgartner, T., Metzler, S., et al., 2010. Oxytocin enhances amygdala-dependent, socially reinforced learning and emotional empathy in humans. J. Neurosci. 30, 4999-5007.

Insel, T.R., Young, L.J., 2001. The neurobiology of attachment. Nat. Rev. Neurosci. 2, 129-136.

Kenyon, A.R., Alvares, G.A., Hickie, I.B., Guastella, A.J., 2013. The effects of acute arginine vasopressin administration on social cognition in healthy males. J. Horm. 2013, 1-4.

Kim, S.-J., Young, L.J., Gonen, D., Veenstra-VanderWeele, J., Courchesne, R., Courchesne, E., et al., 2002. Transmission disequilibrium testing of arginine vasopressin receptor 1A (AVPR1A) polymorphisms in autism. Mol. Psychiatry 7, 503-507.

Knafo, A., Israel, S., Darvasi, A., Bachner-Melman, R., Uzefovsky, F., Cohen, L., et al., 2008. Individual differences in allocation of funds in the dictator game associated with length of the arginine vasopressin 1a receptor RS3 promoter region and correlation between RS3 length and hippocampal mRNA. Genes Brain Behav. 7, 266-275.

Koshimizu, T., Nakamura, K., Egashira, N., Hiroyama, M., Nonoguchi, H., Tanoue, A., 2012. Vasopressin V1a and V1b receptors: from molecules to physiological systems. Physiol. Rev. 92, 1813-1864.

Lim, M.M., Young, L.J., 2006. Neuropeptidergic regulation of affiliative behavior and social bonding in animals. Horm. Behav. 50, 506-517.

Ludwig, M., Tobin, V.A., Callahan, M.F., Papadaki, E., Becker, A., Engelmann, M., Leng, G., 2013. Intranasal application of vasopressin fails to elicit changes in brain immediate early gene expression, neural activity and behavioural performance of rats. J. Neuroendocrinol. 25, 655-667.

Macdonald, K.S., 2012. Sex, receptors, and attachment: a review of individual factors influencing response to oxytocin. Front. Neurosci. 6, 194.

Parker, G., Tupling, H., Brown, L.B., 1979. A parental bonding instrument. Br. J. Med. Psychol. 52, 1-10.

Riem, M.M.E., Bakermans-Kranenburg, M.J., Huffmeijer, R., van Ijzendoorn, M.H., 2013. Does intranasal oxytocin promote prosocial behavior to an excluded fellow player? A 
randomized-controlled trial with Cyberball. Psychoneuroendocrinology 38, 1418-1425.

Rilling, J.K., Demarco, A.C., Hackett, P.D., Chen, X., Gautam, P., Stair, S., et al., 2014. Sex differences in the neural and behavioral response to intranasal oxytocin and vasopressin during human social interaction. Psychoneuroendocrinology 39, 237-248.

Rilling, J.K., DeMarco, A.C., Hackett, P.D., Thompson, R., Ditzen, B., Patel, R., Pagnoni, G., 2012. Effects of intranasal oxytocin and vasopressin on cooperative behavior and associated brain activity in men. Psychoneuroendocrinology 37, 447-461.

Shalev, I., Israel, S., Uzefovsky, F., Gritsenko, I., Kaitz, M., Ebstein, R.P., 2011. Vasopressin needs an audience: neuropeptide elicited stress responses are contingent upon perceived social evaluative threats. Horm. Behav. 60, 121-127.

Shamay-Tsoory, S.G., 2011. The neural bases for empathy. Neuroscientist 17, 18-24.

Shamay-Tsoory, S.G., Abu-Akel, A., Palgi, S., Sulieman, R., Fisher-Shofty, M., Levkoviz, Y., et al., 2013. Giving peace a chance: oxytocin increases empathy to pain in the context of the Israeli-Palestinian conflict. Psychoneuroendocrinology 38, 3139-3144.

Singer, T., Snozzi, R., Bird, G., Petrovic, P., Silani, G., Heinrichs, M., Dolan, R.J., 2008. Effects of oxytocin and prosocial behavior on brain responses to direct and vicariously experienced pain. Emotion 8, 781-791.

Sze, J.A., Gyurak, A., Goodkind, M.S., Levenson, R.W., 2012. Greater emotional empathy and prosocial behavior in late life. Emotion 12, 1129-1140.

Tabak, B.A., 2013. Oxytocin and social salience: a call for gene-environment interaction research. Front. Neurosci. 7, 199.

Taylor, S.E., Saphire-Bernstein, S., Seeman, T.E., 2010. Are plasma oxytocin in women and plasma vasopressin in men biomarkers of distressed pair bond relationships? Psychol. Sci. 21, 3-7.

Theodoridou, A., Penton-Voak, I.S., Rowe, A.C., 2013. A direct examination of the effect of intranasal administration of oxytocin on approach-avoidance motor responses to emotional stimuli. PLOS ONE 8, e58113.

Thompson, E.R., 2007. Development and validation of an internationally reliable short-form of the positive and negative affect schedule (PANAS). J. Cross. Cult. Psychol. 38, 227-242.
Thompson, R.R., George, K., Walton, J.C., Orr, S.P., Benson, J., 2006. Sex-specific influences of vasopressin on human social communication. Proc. Natl. Acad. Sci. U. S. A. 103, 7889-7894.

Uzefovsky, F., Shalev, I., Israel, S., Knafo, A., Ebstein, R.P., 2012. Vasopressin selectively impairs emotion recognition in men. Psychoneuroendocrinology 37, 576-580.

Van IJzendoorn, M.H., Huffmeijer, R., Alink, L.R., Bakermans-Kranenburg, M.J., Tops, M., 2011. The impact of oxytocin administration on charitable donating is moderated by experiences of parental love-withdrawal. Front. Psychol. 2, 258.

Veenema, A.H., 2012. Toward understanding how early-life social experiences alter oxytocin- and vasopressin-regulated social behaviors. Horm. Behav. 61, 304-312.

Veenema, A.H., Neumann, I.D., 2009. Maternal separation enhances offensive play-fighting, basal corticosterone and hypothalamic vasopressin mRNA expression in juvenile male rats. Psychoneuroendocrinology 34, 463-467.

Walum, H., Westberg, L., Henningsson, S., Neiderhiser, J.M., Reiss, D., Igl, W., et al., 2008. Genetic variation in the vasopressin receptor $1 \mathrm{a}$ gene (AVPR1A) associates with pair-bonding behavior in humans. Proc. Natl. Acad. Sci. U. S. A. 105, 14153-14156.

Wang, Z., Young, L.J., De Vries, G.J., Insel, T.R., 1999. Prog. Brain Res. 119, 483-499.

Wassink, T.H., Piven, J., Vieland, V.J., Pietila, J., Goedken, R.J., Folstein, S.E., Sheffield, V.C., 2004. Examination of AVPR1a as an autism susceptibility gene. Mol. Psychiatry 9, 968-972.

Winslow, J.T., Hastings, N., Carter, C.S., Harbaugh, C.R., Insel, T.R., 1993. A role for central vasopressin in pair bonding in monogamous prairie voles. Nature 365, 545-548.

Yirmiya, N., Rosenberg, C., Levi, S., Salomon, S., Shulman, C., Nemanov, L., et al., 2006. Association between the arginine vasopressin 1a receptor (AVPR1a) gene and autism in a family-based study: mediation by socialization skills. Mol. Psychiatry 11, 488-494.

Zhou, Q., Eisenberg, N., Losoya, S.H., Fabes, R.A., Reiser, M., Guthrie, I.K., et al., 2002. The relations of parental warmth and positive expressiveness to children's empathy-related responding and social functioning: a longitudinal study. Child Dev. 73, 893-915. 